\title{
Li-rich giants: A survey based on IRAS colours ${ }^{\star}$
}

\author{
B.V. Castilho ${ }^{1}$, J. Gregorio-Hetem ${ }^{1}$, F. Spite ${ }^{2}$, M. Spite ${ }^{2}$, and B. Barbuy ${ }^{1}$ \\ 1 Universidade de São Paulo - IAG, Depto. de Astronomia, CP 3386 - 01060-970 São Paulo, Brazil \\ ${ }^{2}$ Observatoire de Paris-Meudon, 92195 Meudon Cedex, France
}

Received April 8; accepted April 24, 1997

\begin{abstract}
In a previous work we studied the IRAS colours of known Li-rich red giants and showed that they have flux ratios $F_{12} / F_{25}$ and $F_{25} / F_{60}$ in well defined ranges. By using this result as a selection criterion, we prepared a list of 280 IRAS Point Source candidates to be Li-rich giant stars.

Up to the present we have obtained spectra for $57 \%$ of our target list. We identified five stars showing a strong LiI $670.079 \mathrm{~nm}$ line and six ones with a Li line of medium strength. Most of the candidates show features typical of normal giants having circumstellar dust, as indicated by their IRAS colours.
\end{abstract}

Key words: stars: abundances — infrared: stars — stars: late type

\section{Introduction}

In Gregorio-Hetem et al. (1993) and Castilho (1995) the far-infrared emission of Li-rich giants (LRG) was analyzed, based on data from the IRAS Point Source Catalog for the sample of LRG discovered by Brown et al. (1989, hereafter BSLD). BSLD obtained coudé spectra for 644 giants, and found 9 new LRG $(\log N(\mathrm{Li})>1.8)$, which corresponds to $\sim 1.5 \%$ of their sample of $G-K$ giants. They also found 8 stars with $1.3<\log N(\mathrm{Li})<1.8(\sim 1.2 \%$ of their sample $)$.

In terms of IRAS colours the distribution of LRG is different from that of Li-normal giants. A large number of LRG are found within the "box" $-0.7<[25-12]<-0.3$ and $0.0<[60-25]<0.25$ as shown by Gregorio-Hetem et al. (1993) (these colours are defined by: $\left[\lambda_{i}-\lambda_{j}\right]=$ $\log \left(S_{\lambda_{i}} / S_{\lambda_{j}}\right)$, where $\lambda=12,25$, and $\left.60 \mu \mathrm{m}\right)$.

Following the criterion of flux ratios given above, we have selected 280 candidates from the IRAS PSC and

Send offprint requests to: B.V. Castilho

* Observations collected at the Laboratório Nacional de Astrofísica - LNA, Brazil; Observatoire de Haute Provence OHP, France; European Southern Observatory - ESO, Chile. started an all sky survey of these stars (Barbuy et al. 1995, 1996). In this paper results of this survey are presented.

In Sect. 2 the selection criterion and the observations are described. In Sect. 3 the results are presented and discussed.

\section{Selection of candidates and observations}

Using the "box" in IRAS colours $-0.7<[25-12]<-0.3$ and $0.0<[60-25]<0.25$ (Gregorio-Hetem et al. 1993), 280 candidates to be LRG were selected from the IRAS PSC I and II (Joint IRAS Science Working Group 1985 \& 1988).

Spurious objects were avoided by retaining only sources having good quality IRAS measurements. All sources previously identified as early-type stars, extragalactic or non-stellar objects were not considered.

The observations were collected at the Laboratório Nacional de Astrofísica (LNA), Brazil; European Southern Observatory (ESO), Chile and Observatoire de Haute Provence (OHP), France.

The northern hemisphere observations were developed at OHP by using the AURELIE high resolution spectrometer (Gillet et al. 1994) at the $1.52 \mathrm{~m}$ coudé Telescope. The observations cover the spectral range $640-680 \mathrm{~nm}$, with spectral resolution of $0.02 \mathrm{~nm}$.

The southern candidates were observed at LNA and ESO. At LNA spectra were obtained at the coudé focus of the $1.6 \mathrm{~m}$ telescope, using a GEC CCD covering the spectral range $663-677 \mathrm{~nm}$, with spectral resolution of $0.3 \AA$. The ESO observations were collected at the $1.52 \mathrm{~m}$ ESO telescope with the Boller \& Chivens Spectrograph and a CCD FA2K, using the grating \#26, which gives a resolution of $\sim 1 \AA$ in the spectral range $530-730 \mathrm{~nm}$.

We have observed 158 IRAS sources in the period 19941996. In the case of IRAS sources associated to two stars, both were observed so that the total sample includes 164 stars. 
Table 1. List of observed stars which show high and medium equivalent width of the Li $670.782 \mathrm{~nm}$ line

\begin{tabular}{lcclllll}
\hline \hline IRAS name & $\alpha_{1950}$ & $\delta_{1950}$ & identification & S.T. & $m_{V}$ & $W(\mathrm{Li})$ & $W(\mathrm{Li}) / W(\mathrm{Ca})$ \\
\hline $07559-5859$ & 075554.5 & -585922 & HD 65750, SAO 235638 & M0III & 6.3 & 0.31 & 1.41 \\
$16161-1445$ & 161611.4 & -144510 & HD 146850, SAO 159846 & K3III & 7.2 & 0.37 & 1.32 \\
$18241-1443$ & 182406.6 & -144341 & GCSS557 & SRa & 10.4 & 0.21 & 0.95 \\
$18585-0430$ & 185830.6 & -043039 & HD 176588, SAO 142919 & K2III & 7.1 & 0.27 & 1.00 \\
$19012-0747$ & 190115.6 & -074706 & & & & 0.41 & 1.71 \\
\hline \hline $00483-7347$ & 004818 & -734700 & & & & 0.10 & 0.53 \\
$06215-0902$ & 062133.9 & -090226 & HD 44889, SAO 133209 & K0 & 8.2 & 0.18 & 0.60 \\
$10204-6135$ & 102026.0 & -613551 & HD 90082, SAO 250932 & M3 & 7.5 & 0.20 & 0.83 \\
$11024-6241$ & 110227.0 & -624126 & HD 96195, SAO 251247 & K5 & 8.0 & 0.17 & 0.61 \\
$19038-0026$ & 190349.9 & -002604 & & & & 0.13 & 0.77 \\
$19049-0234$ & 190459.5 & -023432 & HD 178168 & K5 & 9.0 & 0.12 & 0.53 \\
\hline
\end{tabular}

We use "GCSS" to identify an object in the General Catalog of S stars (Stephenson 1976).

\section{Results}

We have observed about half of the list of candidates, and found 5 new LRG.

Among the 164 observed stars we have found: 5 Lirich giants; 6 with a moderate Li line; 20 normal giants showing a weak Li line, and 56 showing no Li line; 54 stars present strong molecular $\mathrm{TiO}$ bands; and 23 remaining ones correspond to other kinds of objects. The different groups of objects are described in Appendix A.

We estimate the strength of the $\mathrm{Li}$ line relative to the Ca I $671.768 \mathrm{~nm}$ line. The candidates were classified in three groups, according to the the ratio between the Equivalent Width $(W)$ of these lines: $W(\mathrm{Li}) / W(\mathrm{Ca}) \gtrsim 1$, $0.5 \lesssim W(\mathrm{Li}) / W(\mathrm{Ca})<1$, and $W(\mathrm{Li}) / W(\mathrm{Ca})<0.5$ are considered strong, moderate and weak, respectively.

\subsection{Li-rich giants}

The stars showing strong and moderate Li line are listed in Table 1, where are given: the IRAS name; equatorial coordinates; other identification; the visual magnitude and the spectral type when available; the equivalent width (in $\AA$ ) of the Li I $670.782 \mathrm{~nm}$ doublet $W(\mathrm{LiI})$; and the ratio $W(\mathrm{LiI}) / W(\mathrm{CaI})$. Comments on individual objects are presented in Appendix A.

It should be noted that the equivalent width of the $\mathrm{H} \alpha$ line for all these stars is $\sim 1 \AA$, in agreement with results found for G5 - M5 red giants. In Figs. 1 and 2 we show the spectra obtained for stars in Table 1. For one of them (HD 146850) we carried out a detailed analysis based on high resolution spectroscopy (Castilho et al. 1995). Files containing the spectra in the Li I line region for all these stars, and an échelle spectrum of HD 146850, are available in CD ROM (Castilho et al. 1997).

The lithium is expected to be strongly diluted in giant stars according to the standard models. Observations show that in disk stars (BSLD) and globular clusters (e.g.
Pasquini \& Molaro 1996) the Li abundance for most of the giants is even lower than expected from the models.

$\mathrm{Li}$ abundances 200 times larger than the mean value indicate that, either (a) in some stars the original $\mathrm{Li}$ is not destroyed, or (b) there should be a process of Li production during the evolution of the star.

Smith \& Lambert $(1989,1990)$ have shown that evolved (AGB) red giants could produce Li during particular phases of their evolution. Theoretical predictions for such production of $\mathrm{Li}$ in intermediate mass stars $\left(3-7 M_{\odot}\right)$ were presented by Sackmann \& Boothroyd (1992). Sackman \& Boothroyd (1997) using deep circulation computations extend the mass range of Li production to stars of $1-3 M_{\odot}$.

Some groups of LRG are found in other locations of the IRAS colours diagram, suggesting that they are following tracks in this diagram, probably caused by mass loss (Castilho 1995; de la Reza et al. 1996). In order to test this hypothesis, other regions in the IRAS diagram should be investigated such as the region between the $l o-$ cus studied by us and the T Tauri locus (Gregorio-Hetem et al. 1992). A tentative list of IRAS sources in this region has 400 sources, but the number of identified stars is very poor.

Except for the high Li abundance, the LRG are normal red giants. No correlation was found between mass, rotation or ${ }^{12} \mathrm{C} /{ }^{13} \mathrm{C}$ ratio and $\mathrm{Li}$ abundance (da Silva et al. 1995; de Medeiros et al. 1996). This fact and their far infrared emission features suggest that we may be observing not a unique class of stars, but a short phase of the stellar evolution of ordinary stars, when Li is created (Sackmann \& Boothroyd 1997).

Detailed analyses for the present sample of LRGs, based on high resolution spectroscopy, will be presented elsewhere. Also, the measurement of B and Be would be of great interest (Sackmann \& Boothroyd 1997).

Acknowledgements. We are grateful to Suzanne Huille for kindly carrying out the observations during two of our runs 


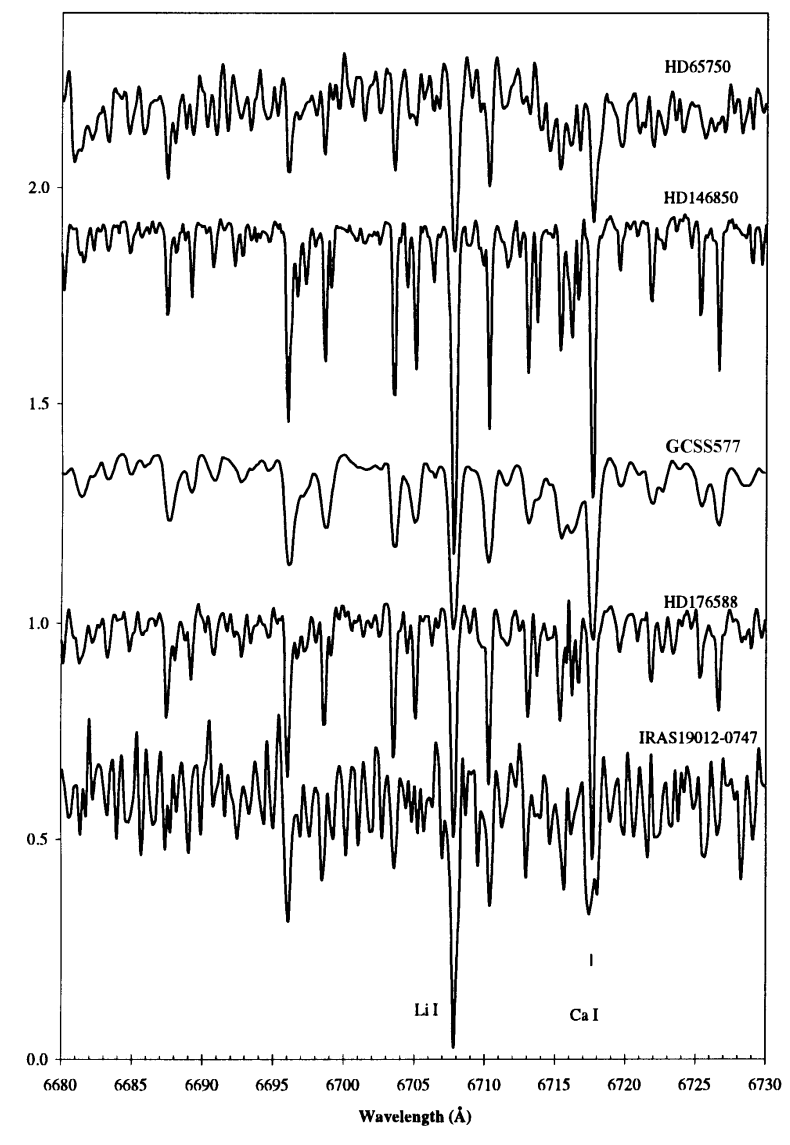

Fig. 1. Spectra of the five giants showing large Li-line. Differences in line widths are due to spectral resolution, see Sect. 2

at OHP. This work benefited from the SIMBAD database operated by Centre des Données Stellaires (CDS) in Strasbourg. We acknowledge partial financial support by CNPq, Fapesp (Brazil) and CNRS (France). BC and JGH acknowledge the CNPq PhD (Proc. No. 840417/97-7) and Post-Doc (Proc. No. 300267/92-4) fellowships.

\section{Appendix}

\section{A. Description of observed stars}

\section{A.1. Normal giants}

In recent work it has been shown that the mass loss rate in the red giant branch appears to be higher than previously thought (Zuckerman et al. 1995). Most of the objects observed in our survey are normal giants showing a farinfrared emission typical of circumstellar dust. In Table 2 we list the 20 normal giants showing a faint $\mathrm{Li}$ line. The 57 normal stars, with no Li line, are listed in Table 3.

de la Reza et al. (1997) argue that the presence of dust around normal-Li giants is connected to a $\mathrm{Li}$ depletion occurring faster than the shell dissipation. The normal giants are located apart from the LRG, the latter being

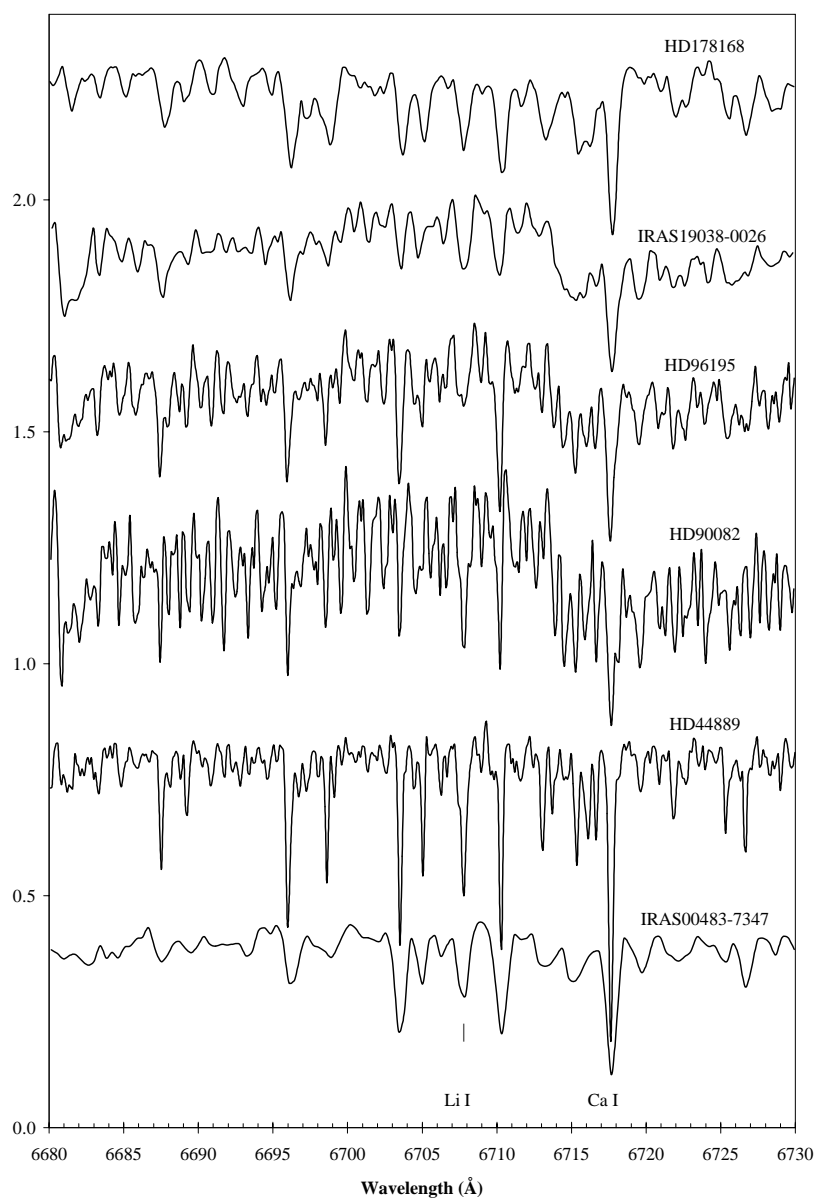

Fig. 2. Spectra of the six giants showing mid strength Li-line. Differences on line widths are due to spectral resolution, see Sect. 2

redder. We note that in the IRAS diagram "box" surveyed in the present work this effect is not observed.

\section{A.2. Cool giants}

A large group of stars with strong molecular bands is listed in Table 4 . The bandheads are mainly at 665.1,668.0, and $671.7 \mathrm{~nm}$, corresponding to $\mathrm{TiO}$ bands.

\section{A.3. Other objects}

A few stars, listed in Table 5, show features not typical of the groups above discussed and we classified them as spurious objects. Most of them show a large $\mathrm{H} \alpha$ absorption line, that could represent early-type stars. We have detected 4 stars with faint $\mathrm{H} \alpha$ emission line, which we tentatively classify as possible weak $\mathrm{T}$ Tauri stars. We also detected 2 Carbon stars. 
Table 2. List of stars showing features of normal giants, with low equivalent width of the $\mathrm{Li}$ line

\begin{tabular}{|c|c|c|c|c|c|}
\hline$\overline{\text { IRAS name }}$ & identificatiol & $\overline{\text { S.T. }}$ & $\overline{m_{V}}$ & $\overline{W(\mathrm{Li}}$ & $W(\mathrm{Li}) / W(\mathrm{Ca})$ \\
\hline$\overline{01504+5953}$ & DO24938 & M3 & & 0.05 & 0.32 \\
\hline $02401+8139$ & SAO432 & $\mathrm{K} 2$ & 8.5 & 0.10 & 0.43 \\
\hline $03396+5111$ & SAO24181 & K7 & 8.5 & 0.08 & 0.36 \\
\hline $05505+0932$ & SAO113234 & $\mathrm{K} 2$ & 8.1 & 0.03 & 0.13 \\
\hline $07365-1923$ & & & & 0.10 & 0.20 \\
\hline $12100-6952$ & S Mus & & & 0.01 & 0.05 \\
\hline $13027-6024 \mathrm{~A}$ & & & & 0.09 & 0.08 \\
\hline $14262-5805$ & CGCS2163 & & & 0.04 & 0.33 \\
\hline $16583-0408$ & BSC6318 & K4I & 4.8 & 0.06 & 0.28 \\
\hline $17464+6739$ & & & & 0.01 & 0.25 \\
\hline $18232+0800$ & BSC6902 & G8I & 5.6 & 0.01 & 0.05 \\
\hline $18416+0232$ & & & & 0.03 & 0.30 \\
\hline $18453+1949$ & SAO104107 & K3 & 8.0 & 0.06 & 0.23 \\
\hline 19019-1039A & & & & 0.01 & 0.06 \\
\hline $19246+2826$ & DY Cyg & & & 0.01 & 0.48 \\
\hline $19425+4939$ & $\mathrm{SAO} 48778$ & K0 & 7.6 & 0.06 & 0.32 \\
\hline $19575+7618$ & SAO9582 & MA & & 0.03 & 0.18 \\
\hline $20550+6418$ & & & & 0.06 & 0.29 \\
\hline $21121+4920$ & DO39334 & & & 0.03 & 0.25 \\
\hline $21196+4648$ & SAO50754 & K3I & 7.8 & 0.01 & 0.35 \\
\hline
\end{tabular}

We use "CGCS" to identify an object in the General Catalogue of Cool Carbon Stars (Stephenson 1989, also designed by GCGCS); "DO": Dearborn Observatory Catalogue of Faint Red Stars; "BSC": Bright Star Catalogue.

Table 5. Other objects

\begin{tabular}{llccl}
\hline \hline IRAS name & identification & S.T. & $m_{V}$ & note \\
\hline $05142+7910$ & SAO 5496 & F8 & 5.1 & $\mathrm{a}$ \\
$06444+0038$ & CGCS 580 & & & $\mathrm{c}$ \\
$06489-0511$ & CGCS 597 & & & $\mathrm{c}$ \\
$08197-3447$ & CGCS 1143 & & & $\mathrm{~b}$ \\
$08217-4518$ & CGCS 1158 & & & $\mathrm{~d}$ \\
$08249-4425 \mathrm{~B}$ & & $\mathrm{a}$ \\
$13301-5516 \mathrm{D}$ & ER Cen & & & $\mathrm{a}$ \\
$15053-5322$ & CGCS 2214 & & $\mathrm{~d}$ \\
$17275-1016$ & & & $\mathrm{a}$ \\
$18339-1323 \mathrm{~A}$ & & $\mathrm{~b}$ \\
$18339-1323 \mathrm{~B}$ & & & $\mathrm{a}$ \\
$18587+0947$ & & & $\mathrm{a}$ \\
$19019-1039 \mathrm{~B}$ & & & $\mathrm{a}$ \\
$19136+2127$ & & & $\mathrm{~d}$ \\
$19160+4641$ & & & $\mathrm{~d}$ \\
$19235+1054$ & & & $\mathrm{~d}$ \\
$19263+1214$ & & & $\mathrm{a}$ \\
$19337+1620$ & & & $\mathrm{a}$ \\
$19431+3014$ & & & $\mathrm{a}$ \\
$19572+5157 \mathrm{~A}$ & CM Cyg & & $\mathrm{b}$ \\
$19572+5157 \mathrm{~B}$ & CM Cyg N & & $\mathrm{d}$ \\
$21270+4100$ & & $\mathrm{~b}$ \\
$23481+6154$ & & & $\mathrm{a}$ \\
\hline
\end{tabular}

Notes: a) large $\mathrm{H} \alpha$ absorption, b) $\mathrm{H} \alpha$ emission, c) Carbon star, d) other.

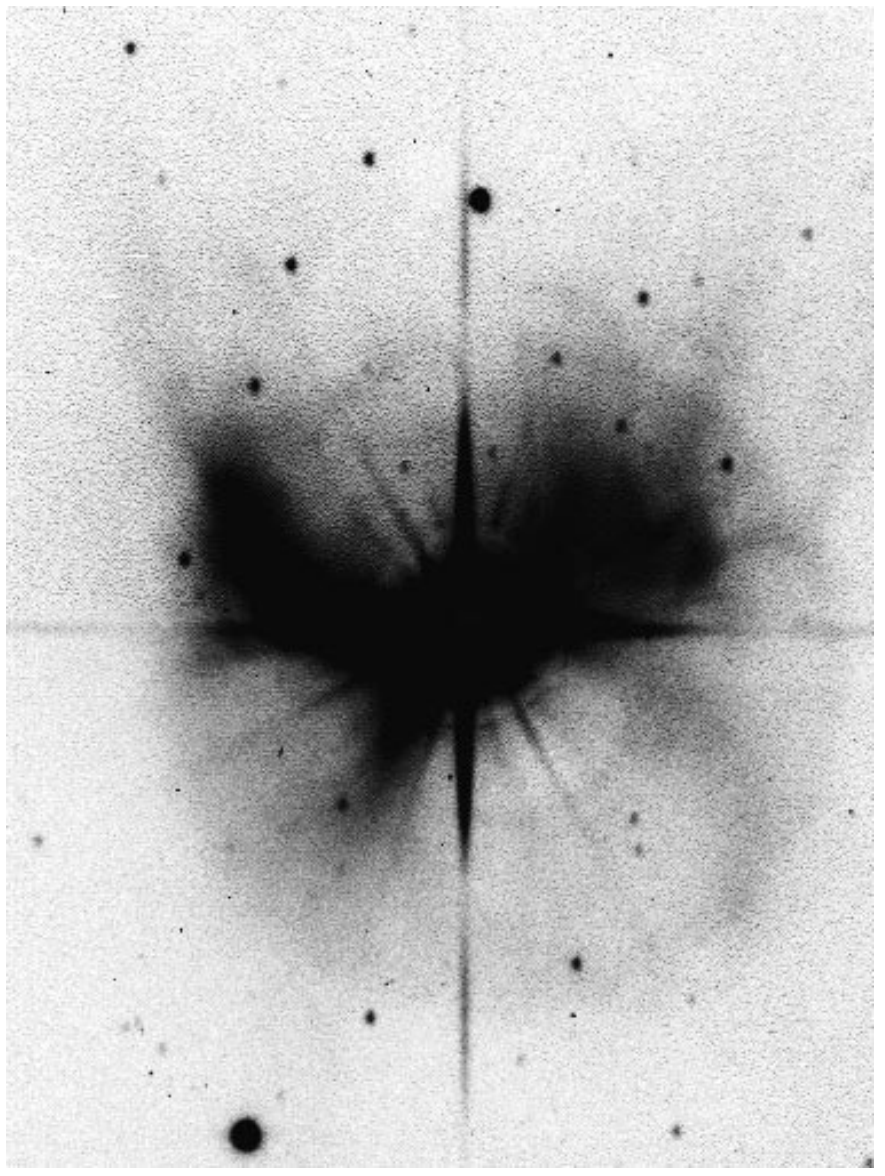

Fig. 3. $I$ band image of HD 65750, showing the large envelope $\left(3^{\prime} \times 5^{\prime}\right)$. North is up and West is to right

\section{A.4. Comments on individual objects}

IRAS 16161 - 1445 (HD 146850) - In Castilho et al. (1995) we carried out a detailed analysis of this star and found $\log N(\mathrm{Li})=1.6,[\mathrm{Fe} / \mathrm{H}]=-0.3$ and solar ratios of $\alpha$ elements $\mathrm{O}, \mathrm{Mg}, \mathrm{Ca}$, Ti relative to Fe. This star is a typical disk star.

IRAS 07559 - 5859 (HD 65750) - This $5 M_{\odot}$ (Pesce et al. 1989) oxygen-rich star is very interesting because of its large $(3-4$ arcmin $)$ dust and gas envelope. Reimers (1977) estimated a $2-410^{-7} M_{\odot} /$ year mass loss rate, and an envelope mass of $\sim 0.7 M_{\odot}$. Witt \& Rogers (1991) estimated that this mass loss rate is not enough to form the observed envelope and they proposed that a past and more efficient mass loss event occurred about 32000 years ago. Its evolutionary status is not well defined due to the large uncertainties in the mass and luminosity determinations. Probably HD 65750 is at the base of the AGB.

The presence of this large envelope confirms our previous suggestion (Gregorio-Hetem et al. 1993) that the Lirich giants are associated with circumstellar dust and gas envelopes emitting mostly in the infrared, and that this 
characteristic could be related with the high $\mathrm{Li}$ abundances (Castilho 1995; de la Reza et al. 1996).

In Fig. 3 we present an $I$ band CCD image of HD 65750, showing its large envelope (IC 2220). The image was obtained with a $60 \mathrm{~s}$. exposure at the $60 \mathrm{~cm} \mathrm{B \& C}$ telescope at LNA (Brazil).

IRAS 10204 - 6135 (HD 90082) - Although the Li line doublet in this star is comparable to the Ca I line $(\lambda 671.7 \mathrm{~nm})$, the molecular lines in this region do not permit a good estimation of the $W(\mathrm{Li})$.

IRAS 19012 - 0747 and IRAS $19038-0026$ - We found no identification for the optical counterparts of these two IRAS sources and no previous work on then. Both seem to be K stars. The $W(\mathrm{Li}) / W(\mathrm{Ca})$ ratio for IRAS $19012-0747$ is the largest of our sample.

IRAS 18241 - 1443 (GCSS 577) - Chen \& Kwok (1993) used this star in their circumstellar material study. It was identified as a S star by Kershbaum \& Hron (1992). Its low resolution IRAS spectrum shows no peculiar features in the range $8-22 \mu \mathrm{m}$ (Olnon \& Raimond 1986).

\section{References}

Barbuy B., Castilho B.V., Gregorio-Hetem J., 1995, in Physical Processes in Astrophysics, Roxburg I.W. and Masnou J.-L. (eds.). Springer-Verlag, p. 191

Barbuy B., Castilho B.V., Gregorio-Hetem J., 1996, Mem. Soc. Astr. It. 66, 413

Brown J.A., Sneden C., Lambert D.L., Dutchover E., 1989, ApJS 71, 293 (BSLD)

Castilho B.V., 1995, Master Degree Dissertation, Depto. de Astronomia, IAG/USP

Castilho B.V., Barbuy B., Gregorio-Hetem J., 1995, A\&A 297, 503

Castilho B.V., Gregorio-Hetem J., Barbuy B., 1997, J. Astron.
Data 3, 1 (CD-ROM)

Chen P.S., Kwok S., 1993, ApJ 416, 769

da Silva L., de la Reza R., Barbuy B., 1995, ApJ 448, L41

de la Reza R., Drake N., da Silva L., 1996, ApJ 456, L115

de la Reza R., Drake N., da Silva L., Martin E., 1997, ApJ 482, L77

de Medeiros J.R., Melo C.H.F., Mayor M., 1996, A\&A 309, 465

Gillet D., Burnage R., Kohler D., et al., 1994, A\&AS 108, 181

Gregorio-Hetem J., Lépine J.R.D., Torres C.A.O., Quast G.R., de la Reza R., 1992, AJ 103, 549

Gregorio-Hetem J., Castilho B.V., Barbuy B., 1993, A\&A 268, L25

IRAS Point Source Catalog - Joint IRAS Science Working Group, US Gov. Printing Office, 1985

IRAS Point Source Catalog II - Joint IRAS Science Working Group, Infrared Processing and Analysis Center, 1988, in The Astronomical Data Center CD-ROM, Selected Astronomical Catalogs, Vol. 1

Kerschbaum F., Hron J., 1992, A\&A 263, 97

Olnon F.M., Raimond E., IRAS Science Team, 1986, A\&AS 65,607

Pasquini L., Molaro P., 1996, A\&A 307, 761

Pesce J.E., Stencel R.E., Doggett J., Walter F.M., Whitelock P.A., Dachs J., 1989, in Evolution of Peculiar Red Giants Stars, IAU Coll. 106, Johnson H.R., Zuckerman B. (eds.). Camb. Un. Press, p. 55

Reimers D., 1977, A\&A 51, 185

Sackmann I.-J., Boothroyd A., 1992, ApJ 392, L71

Sackmann I.-J., Boothroyd A., 1997, ApJ (submitted)

Smith V., Lambert, 1989, ApJ 345, L75

Smith V., Lambert, 1990, ApJ 361, L69

Stephenson C.B., 1976, Publ. Warner Swasey Obs. 2, 21

Stephenson C.B., 1989, Publ. Warner Swasey Obs. 3, 53

Zuckerman B., Kinn S.S., Liu T., 1995, ApJ 446, L79

Witt A.N., Rogers C., 1991, PASP 103, 415 
Table 3. List of normal stars with no Li line

\begin{tabular}{|c|c|c|c|}
\hline$\overline{\overline{\text { IRAS name }}}$ & "identification & $\overline{\text { S.T. }}$ & $\overline{m_{V}}$ \\
\hline$\overline{01068+5428}$ & SAO 2204 & K0 & 7.0 \\
\hline $03421+0146$ & SAO 111338 & $\mathrm{~K} 2$ & 8.0 \\
\hline $03557+0118$ & SAO 111532 & K5 & 7.2 \\
\hline $05074-0037$ & SAO 131834 & K0 & 6.3 \\
\hline $05461-0148$ & SAO 132556 & K2 & 7.7 \\
\hline $07150-4034$ & SAO 218604 & G0 & 7.3 \\
\hline 07310-1918 & SAO 153062 & K0 & 5.0 \\
\hline $08158-3554$ & SAO 199051 & K5 & 6.5 \\
\hline $08175-4602$ & SAO 219783 & K0 & 7.0 \\
\hline \multicolumn{4}{|l|}{$08389-4145$} \\
\hline $09184-4457$ & SAO 221077 & K0I & 6.5 \\
\hline \multirow{2}{*}{\multicolumn{4}{|c|}{$09337-5301$}} \\
\hline & & & \\
\hline $11418-5809$ & SAO 239336 & $\mathrm{~K} 2$ & 7.5 \\
\hline $12392-5429$ & SAO 240165 & K5I & 6.5 \\
\hline \multicolumn{4}{|l|}{$12401-6224$} \\
\hline \multicolumn{4}{|l|}{$13027-6024 \mathrm{~B}$} \\
\hline $13086-5643$ & CV Cen & & \\
\hline $13301-5516 \mathrm{~B}$ & ER Cen & & \\
\hline $13301-5516 \mathrm{C}$ & ER Cen & & \\
\hline \multicolumn{4}{|l|}{$13399-5605$} \\
\hline $13432-1210$ & SAO 158152 & K0 & 5.7 \\
\hline $14121-5155$ & CGCS2151 & & \\
\hline $14130-5305$ & SAO 241582 & K5 & 7.7 \\
\hline $14244-5448$ & SAO 241733 & MA & 8.5 \\
\hline \multicolumn{4}{|l|}{$14311-5311$} \\
\hline \multicolumn{4}{|l|}{$14359-5855$} \\
\hline $16094-4600$ & GCSS502 & A & \\
\hline \multicolumn{4}{|l|}{$16103-5321$} \\
\hline $16111-3253$ & SAO 207480 & K0I & 6.0 \\
\hline $16255-5738$ & SAO 243874 & K0 & 6.0 \\
\hline $16553-1417$ & SAO 160195 & $\mathrm{~K} 2$ & 7.2 \\
\hline \multicolumn{4}{|l|}{$17158+1323$} \\
\hline $17388-4333$ & SAO 228311 & K2 & 7.3 \\
\hline $17402+5150$ & SAO 30522 & K0 & 6.0 \\
\hline $17477-2146$ & SAO 185836 & K5 & \\
\hline $17499-1352$ & GCVS9795 & & \\
\hline $17547+0012$ & SAO 122953 & K5 & 8.9 \\
\hline $18048-0636$ & & & \\
\hline \multicolumn{4}{|l|}{$18073+0032$} \\
\hline $18120-0237$ & SAO 142176 & K5 & 7.8 \\
\hline $18383+0708$ & DO5000 & K5 & 9.0 \\
\hline \multicolumn{4}{|l|}{$18405-1732$} \\
\hline \multicolumn{4}{|l|}{$18420-1834$} \\
\hline $18500+1428$ & SAO 104203 & K0 & 6.5 \\
\hline $19049+1702$ & & & \\
\hline $20001+4358$ & SAO 49105 & M0 & 7.2 \\
\hline \multicolumn{4}{|l|}{$20027+2534$} \\
\hline $20035-0413$ & SAO 144062 & K0 & 6.5 \\
\hline \multicolumn{4}{|l|}{$20052+3551$} \\
\hline \multicolumn{4}{|l|}{$20227+5605$} \\
\hline $20375+2925$ & DO19235 & & \\
\hline $21181+5406$ & CCS3011 & & \\
\hline $21217-8501$ & SAO 258899 & $\mathrm{~K} 2$ & 6.4 \\
\hline $22209-0726$ & SAO 146062 & G6I & 6.0 \\
\hline $23579+5916$ & BSC9079 & G9I & 6.1 \\
\hline
\end{tabular}

We use "GCVS" to identify an object in the General Catalog of variable stars.
Table 4. List of the candidates with spectra showing $\mathrm{TiO}$ bands

\begin{tabular}{|c|c|c|c|c|}
\hline IRAS name & identification & S.T. & $m_{V}$ & note \\
\hline $00135+4736$ & DO23016 & & & $\mathrm{ab}$ \\
\hline $06262+0727$ & & & & $a b$ \\
\hline $06319+0500$ & SAO 114079 & MB & 7,1 & $\mathrm{c}$ \\
\hline $07081-2248$ & SAO 173118 & M & & $a b$ \\
\hline $07417-3343$ & SSSC0741-337 & & & $a b$ \\
\hline $08194-4509$ & & & & $\mathrm{a}$ \\
\hline $08234-3536$ & GCSS 311 & & & $\mathrm{~b}$ \\
\hline $08249-4425 \mathrm{~A}$ & & & & $a b$ \\
\hline $08346-5626$ & & & & $\mathrm{~b}$ \\
\hline $08577-4003$ & & & & $\mathrm{~b}$ \\
\hline $10109-5357$ & & & & $\mathrm{a}$ \\
\hline $10551-5250$ & GCSS 418 & & & $\mathrm{a}$ \\
\hline $11252-5510$ & & & & $a b$ \\
\hline $11396-5547$ & UMSA & A3III & & $\mathrm{a}$ \\
\hline $11516-3935$ & UMSA & M3III & & $\mathrm{a}$ \\
\hline $12200-5620$ & & & & $\mathrm{a}$ \\
\hline $12454-7411$ & & & & $\mathrm{a}$ \\
\hline $13057-7115$ & & & & $\mathrm{a}$ \\
\hline 13301-5516A & ER Cen & & & $\mathrm{c}$ \\
\hline $13552-5529$ & & & & $\mathrm{a}$ \\
\hline $13575-5529$ & & & & $\mathrm{c}$ \\
\hline $14066-5657$ & & & & $\mathrm{a}$ \\
\hline $14244-5448$ & SAO 241733 & MA & 8.5 & $a b$ \\
\hline $14528-5134$ & & & & $a b$ \\
\hline $15353-6325$ & & & & $\mathrm{c}$ \\
\hline $15363-4918$ & & & & $\mathrm{c}$ \\
\hline $15591+8313$ & SAO 2638 & & 9.0 & $\mathrm{a}$ \\
\hline $16166-5426$ & & & & $\mathrm{c}$ \\
\hline $16237-5627$ & & & & $\mathrm{~b}$ \\
\hline $16345+0728$ & & & & $\mathrm{a}$ \\
\hline $17050+1449$ & DO15795 & & & $a b$ \\
\hline $17167+1517$ & SAO 102733 & M & 9.1 & $\mathrm{~b}$ \\
\hline $17283-4240$ & & & & $\mathrm{c}$ \\
\hline $17462-0907$ & $2 \mu \mathrm{I} 10380$ & & & $\mathrm{c}$ \\
\hline $18060-0740$ & & & & $\mathrm{a}$ \\
\hline $18160-2636$ & & & & $\mathrm{a}$ \\
\hline $18338+0911$ & DO4926 & & & $\mathrm{a}$ \\
\hline $18405-1732$ & & & & $\mathrm{c}$ \\
\hline $19043+2021$ & & & & $a b c$ \\
\hline $19066-0624$ & & & & $a b$ \\
\hline $19136+0423$ & & & & $\mathrm{~b}$ \\
\hline $19316+1636$ & & & & $\mathrm{a}$ \\
\hline $19468+4019$ & & & & $\mathrm{c}$ \\
\hline $19490+3706$ & V1294 Cyg & & & $\mathrm{c}$ \\
\hline $19579+2518$ & & & & $a b$ \\
\hline $20061+4131$ & V426 Cyg & & & $\mathrm{a}$ \\
\hline $20062+0451$ & SAO 125493 & M0 & 9.0 & $\mathrm{a}$ \\
\hline $20283+7414$ & DO19235 & & & $a b$ \\
\hline $20333+3127$ & & & & $\mathrm{~b}$ \\
\hline $20447+3808$ & & & & $a b$ \\
\hline $21440+5113$ & & & & $a b$ \\
\hline $22131+5557$ & & & & $\mathrm{c}$ \\
\hline $23115+5651$ & & & & $\mathrm{ab}$ \\
\hline $23591+6154$ & DO 43982 & & & $a b$ \\
\hline
\end{tabular}

We use "SSSC" to identify an object in the IRAS Small Scale Structure Catalog; "UMSA": University of Michigan Spectral Atlas; " $2 \mu$ I": Two Micron Sky Survey Improved.

Notes: Detected spectral lines: a) $\mathrm{H} \alpha$, b) Ca I, c) Li I. 